\title{
Tokamak NOVA-UNICAMP Recent Results
}

\author{
A.M. Daltrini, M. Machida, M.J.R. Monteiro, and C. O. Kaminishikawahara \\ Instituto de Física "Gleb Wataghin", Universidade Estadual de Campinas, \\ C. P. 6165, 13083-970, Campinas, SP, Brazil
}

Received on 26 June, 2001

\begin{abstract}
Tokamak NOVA-UNICAMP is a small tokamak with iron core and conducting shell stabilization built to study plasma-wall interaction and optical diagnostic development. Characteristic plasma behavior was the appearance of $2-3 \mathrm{kHz}$ spike oscillation during entire discharge with very high X-ray activity which prevent our optical diagnostic development. By modifying capacitance and charging voltage on $\mathrm{OH}$ and vertical field banks, in order to obtain similar discharge current shape, most of $2-3 \mathrm{kHz}$ spikes has been taken out, although some activities still remains to be analyzed. Nevertheless, the high X-ray activity which jeopardize our optical measurements is completely eliminated and time varying parameter as ion temperature is now possible to be followed during whole discharge.
\end{abstract}

\section{Introduction}

The NOVA-UNICAMP tokamak, former NOVA II tokamak [1] from Kyoto University-Japan, is a small machine operating at our Laboratory since 1996. Its main characteristics are: major radius of $30 \mathrm{~cm}$, minor radius of $6 \mathrm{~cm}$, plasma current of $10 \mathrm{kA}$, plasma discharge time of $15 \mathrm{~ms}$, and toroidal magnetic field of 1 T. Other characteristics can be found in [2].

In our recent publication [3] we have pointed out the progress in spectroscopy research, showing also some tokamak results as peaked oscillations observed in spectral emissions and loop voltage signals.

We present now the results in new mode tokamak discharges and the new diagnostics after some modifications in tokamak parameters and improvement in some other diagnostics as Thomson Scattering, $6 \mathrm{~mm}$ microwave interferometer and Langmuir probe.

\section{Old Mode Discharges}

With four spectrometers in different toroidal positions we have observed in old mode discharges, using hydrogen, $2-3 \mathrm{kHz}$ oscillations both in spectroscopy signals as in loop voltage measurement (Fig. 1).

Operations with He plasma did not present such oscillations, indicating more stable plasma. It was also verified by Doppler broadening line measurements that ion temperature in $\mathrm{H}$ plasmas took at least $4 \mathrm{~ms}$ to reach higher values, a increase much slower than observed with He plasma [3].

Other diagnostics as Thomson scattering and microwave interferometer indicated also higher density plasma: higher than $3 \times 10^{13} \mathrm{~cm}^{-3}$ (cutoff) by interferometry and $5.7 \times 10^{13} \mathrm{~cm}^{-3}$ by Thomson scattering. The last one also gave a low electron temperature: $21 \mathrm{eV}$.

In this way, we supposed that the $2-3 \mathrm{kHz}$ oscillations observed were related with high density and low temperature operation.

A very high level of hard x-ray was observed during the whole discharge, jeopardizing spectroscopy measurements, since photomultipliers were much affected.

Oscillations in old mode operation also did not allow particle confinement time determination by $\mathrm{H}$ emissions, since plasma stability was not obtained.

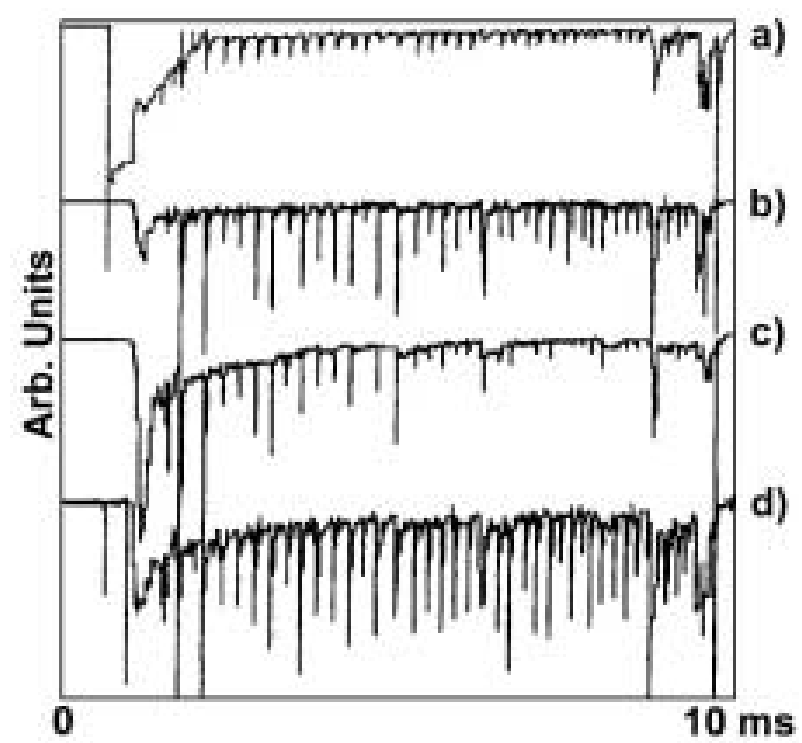

Figure 1. Oscillations of $2-3 \mathrm{kHz}$ peaks, in loop voltage and $H_{\alpha}$ line signals at three toroidal positions: a) Loop Voltage; b) $H_{\alpha}$ at limiter position $\left(0^{\circ}\right)$; c) $H_{\alpha}$ at $90^{\circ}$; d) $H_{\alpha}$ at $180^{\circ}$. 


\section{New Mode Discharges}

After first measurements with tokamak NOVAUNICAMP, showing oscillations in plasma column, high density, low temperature and high x-ray level, we have decided to try some modifications in our tokamak to obtain better discharges.

Next, we present the main changes:

- Changing diode in the circuit for core premagnetization obtaining improvement of the coupling capability for ohmic heating.

- Change in ohmic heating and vertical field capacitance banks to get similar discharges for these banks.

- Improvement in the pre-ionization process applying high voltage $(1 \mathrm{kV})$ between the tungsten filament and a section of the vacuum chamber.

- Cleaning of the vacuum chamber.

- Constant heating of the chamber and discharges with low fields to obtain better vacuum conditions.

To show the influence of the modifications in discharges, plasma current, loop voltage, hard x-ray and $H_{\alpha}$ emission signals can be compared in old mode discharges (Fig. 2) and new mode discharges (Fig. 3).

Analyzing these figures, the first point to be noted is the absence of oscillations in line emissions, $x$-ray and loop voltage signals in the new mode, eliminating one of the problems of old mode discharges.

Other important feature is the almost absence of hard x-ray emission during the discharges, showing a greater modification in ours parameters.

However, plasma current and loop voltage signals do not show yet so good results in this new mode. Loop voltage is too high, indicating that even with the care with vacuum conditions, the level of impurities maybe still high. About the plasma current, although the signals seems to be similar, in new mode discharges we are not able yet to get high plasma current values in the second half of the discharges, while it was able in old mode.

Analysis of other plasma parameters, as can be seen in next section, showed other improvements in new mode discharges:

- High ion temperature in the beginning of the discharge.

- Lower density.

- Higher reproducibility of the discharges.

- Wider range of parameters variations (as density and capacitor bank voltage).

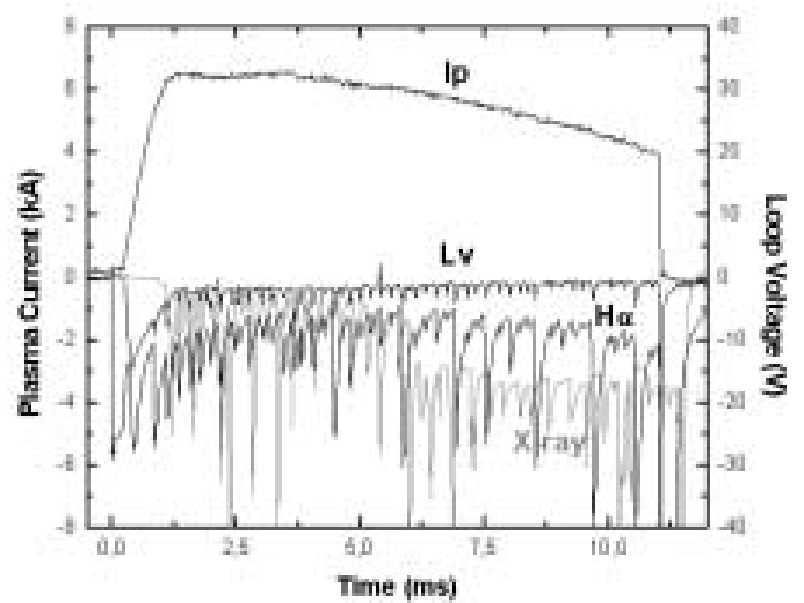

Figure 2. Plasma Current (Ip), Loop Voltage (Lv), $H_{\alpha}$ and hard X-ray signals in old mode discharge (X-ray was taken in a similar shot), where spikes are seen throughout all discharge.

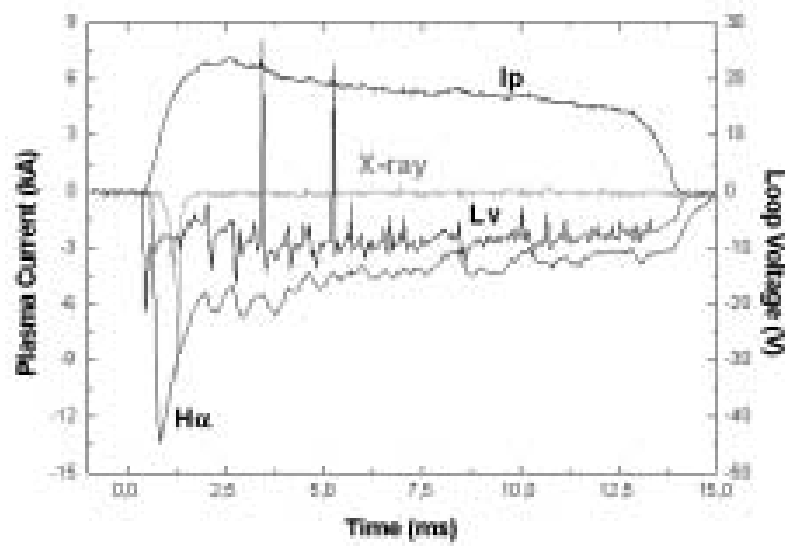

Figure 3. Plasma Current (Ip), Loop Voltage (Lv), $H_{\alpha}$ and hard X-ray signals in new mode discharge, where periodical spikes have been suppressed.

\section{Others Diagnostics Results}

The ion temperature increase in the beginning of the discharges was demonstrated by impurity spectroscopy (Fig. 4).

Calculations of CIII $4647 \AA$ Doppler broadening in the two modes show a quick increase of the temperature in new mode just in the beginning of the discharge, followed by equilibrium, very different than the old case.

Another factor that collaborated with these measurements was the decrease of x-ray intensity, eliminating the noise in photomultipliers and leading to more reliable temperature values.

Microwave interferometry (Fig. 5) also shows an improvement in new configuration, with a lower electron density (Fig. 6).

This result is not so great yet, since still there is a microwave cutoff, and the measurements were done in discharge with low plasma current. However these 
results are better than obtained in discharges with old configuration.

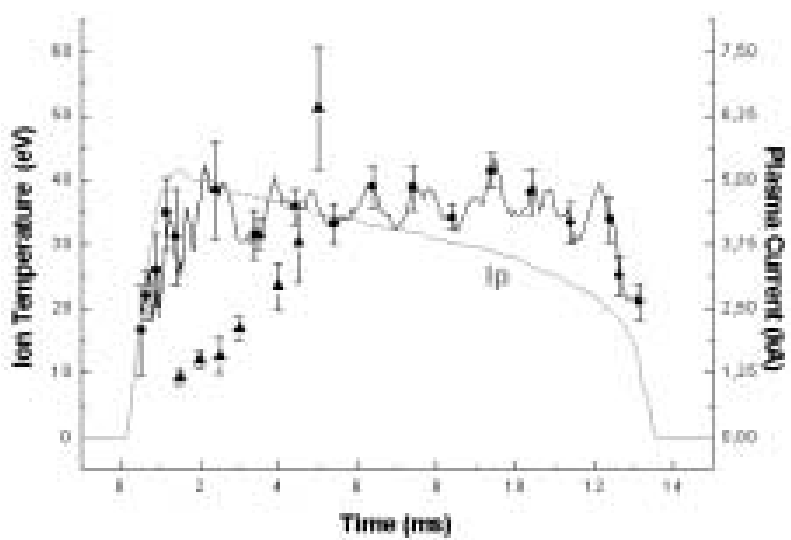

Figure 4. CIII ion temperature in new mode (bold circle) and old mode (triangle) discharges. Plasma current (Ip) is shown in new mode discharge.

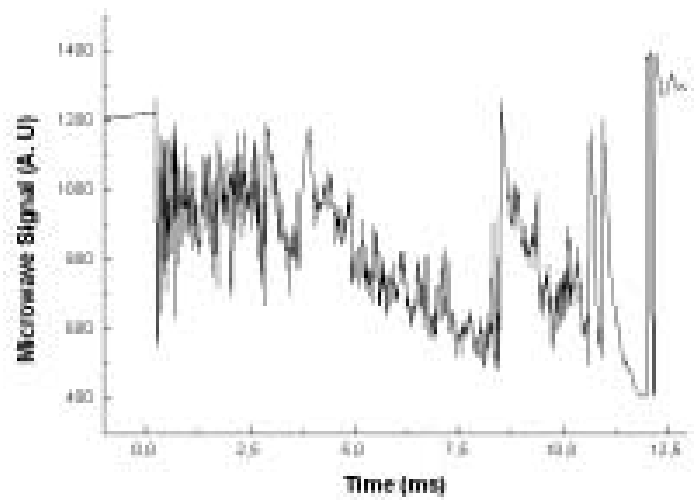

Figure 5. Typical line integrated microwave row signal.

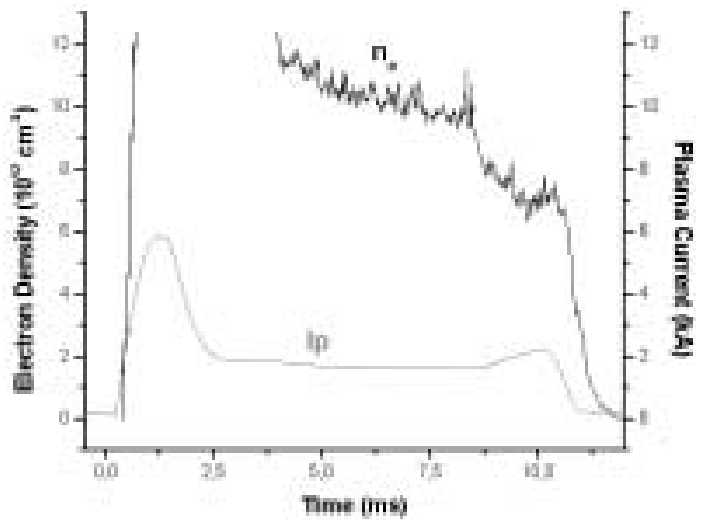

Figure 6. Electron density $\left(\mathrm{n}_{e}\right)$ in a low plasma current (Ip) discharge.

For Langmuir probe measurements at the shadow of the limiter, a sinusoidal sweep voltage control $( \pm$ $100 \mathrm{~V}, 500 \mu \mathrm{s}$ ) has been used. A floated double probe is also available. First measurements also indicated a high density operation. Typical curve I-V of the probe with sweep voltage (low plasma current discharges) can be seen in Fig. 7. Densities as function of probe position are shown in Fig. 8.

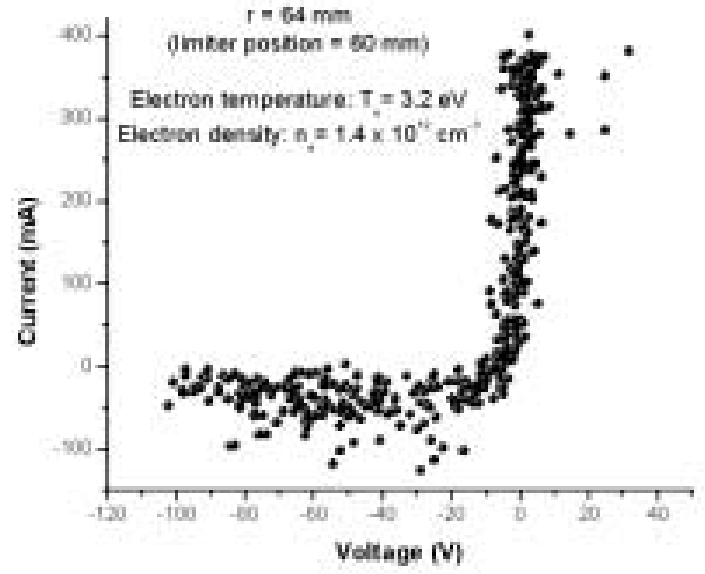

Figure 7. I-V curve for single probe with sweep voltage (500 $\mu \mathrm{s}$ ) in low Ip discharge, taken $4 \mathrm{~mm}$ behind limiter.

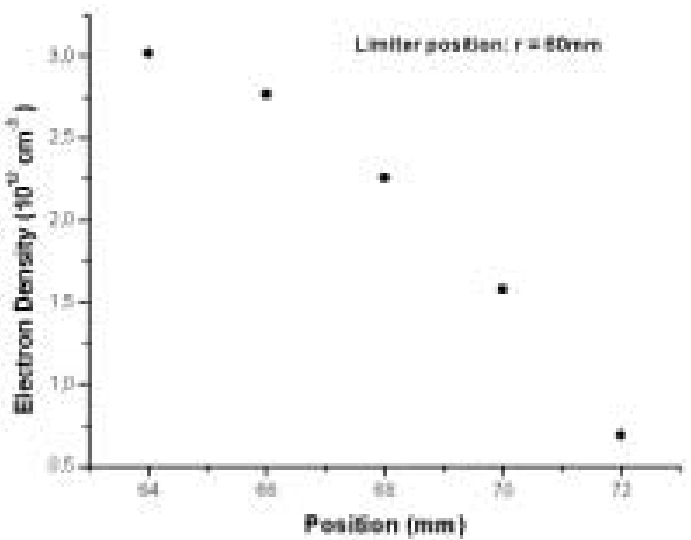

Figure 8. Electron density variation at the shadow of the limiter.

Thomson scattering measurements at new mode discharges has not been done yet but first data will be available in the near future. Multipass system is already mounted at the tokamak (Fig. 9), and a one channel photomultiplier will be used to obtain at least one good condition for scattering measurements.

Multichannel system ( $8 \times 8$ photomultipliers array) is at final tests phase, and will be set new input laser collection optics, obtaining full spatial measurement capability in multipass multispatial scattering.

As for spectroscopy development, 2051 McPherson Spectrometer (Visible) was calibrated with an EPLAB standard tungsten lamp, and VUV Spectrometer will be calibrated by branching-ratio method [4]. First Z effective measurements lead to values between 3 and 7 , and best results will be get with full operation of Thomson scattering and microwave interferometer.

For particle confinement time calculation, measurements are possible now at new mode operation. Lyman and Balmer series will be compared, pointing the advantage in VUV region (no back reflections from walls) and possible molecular effects [5]. 


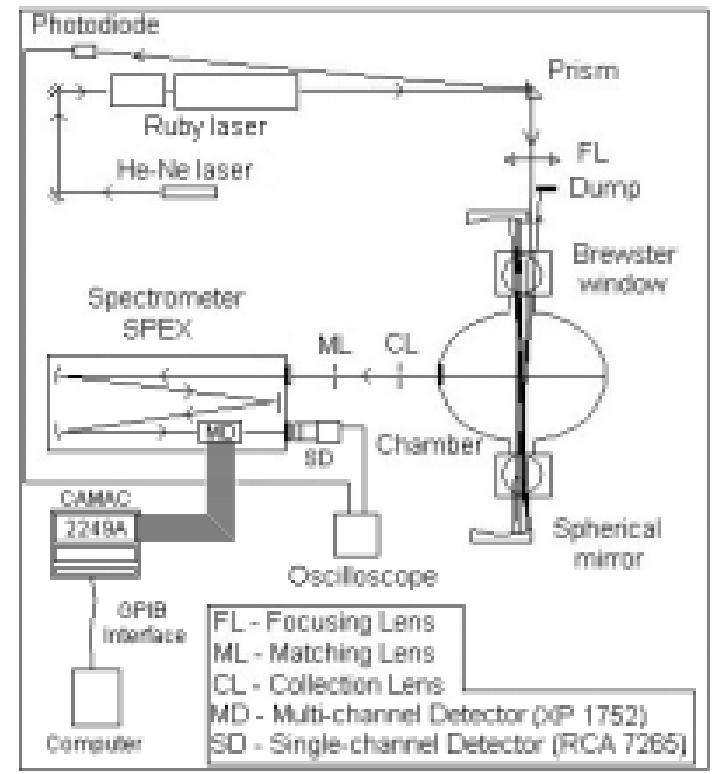

Figure 9. Multipass Thomson scattering system.

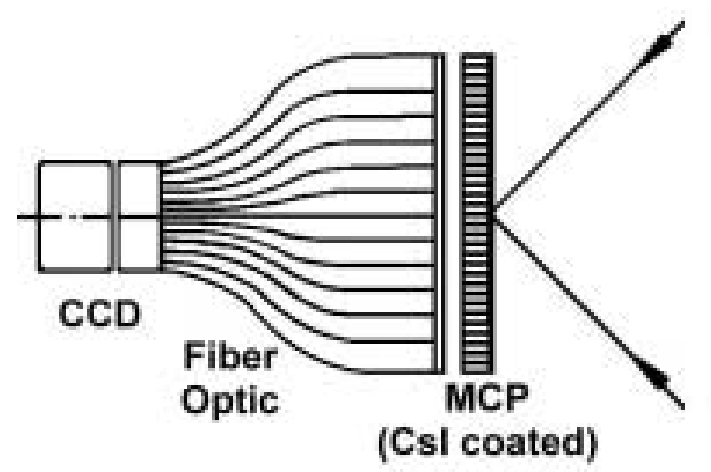

Figure 10. New multichannel assembly for VUV spectroscopy.

Also in spectroscopy detection scheme, an open MCP, using a pulsed power supply with gated widths in the range: $10 \mu \mathrm{s}$ to $1 \mathrm{~ms}$, and a $1024 \times 256$ pixels CCD, Fig. 10, will be set at VUV spectrometer to obtain multichannel detection.
Next step for interferometry will be to obtain signals with lower noise and a new interferometer system with no cutoff.

For measurements at the shadow of the limiter, a movable probe with double and triple probe, is at our plans.

\section{Conclusions}

After first measurements in NOVA-UNICAMP tokamak, modifications were performed to obtain wellbehaved discharges. Although plasma current is not so high and loop voltage needs to decrease. The data in new configuration showed a better plasma condition with higher reproducibly discharges, no $2-3 \mathrm{kHz}$ oscillations, higher plasma temperature at the beginning of the discharge, low hard x-ray level and lower density (still needing improvement). New diagnostics operation and others in development will allow us to obtain and understand better plasma conditions for next works.

\section{Acknowledgements}

We acknowledge Prof. Dr. Masayuki Fukao for constant help in many tokamak modifications and material supporting.

This work is supported by FAPESP - Fundação de Amparo à Pesquisa do Estado de São Paulo, FINEP, CNPq and CAPES.

\section{References}

[1] M. Fukao, K. Mori and T. Tanihara, Plasma Phys. Control. Fusion 33, 199 (1991).

[2] M. Machida et al., "Tokamak NOVA-UNICAMP", published in IV EBFP Proceedings (1996).

[3] A. M. Daltrini, M. Machida and M. J. R. Monteiro, Braz. J. Phys. 31, 496 (2001).

[4] M. Ueda, Braz. J. Phys. 23, 269 (1993).

[5] K. Sawada and T. Fujimoto, J. Appl. Phys. 78, 2913 (1995). 